\title{
Unravelling the heterogeneity of cancer stem cells
}

\section{C6}

at early stages, patients who are likely to develop TKI resistance have SCs that exhibit properties of quiescence כ

\section{Single-cell RNA sequencing} approaches are poised to help unravel the contributions of heterogeneous cell populations within a malignancy to progression, relapse and resistance. However, identifying and analysing the transcriptomics of rare cells, such as cancer stem cells (SCs), is technically challenging. Giustacchini, Thongjuea, et al. have developed a method that allows high-sensitivity detection of somatic mutations and unbiased whole-transcriptome analysis of the same single cell. Application of this technique to chronic myeloid leukaemia (CML) patient samples revealed several distinct CML-SC populations that may have roles in CML progression and resistance to tyrosine kinase inhibitor (TKI) therapy.

The authors first showed that the common Smart-seq 2 single-cell RNA sequencing technique could not sensitively detect the $B C R-A B L$ fusion gene (currently the only definitive marker for CML-SCs) or other common mutations in a CML cell line. By multiplexing $B C R-A B L$ primers with this technique ( $B C R-A B L \mathrm{tSS} 2)$, they were able to improve detection to $100 \%$ in this cell line while still enabling unbiased transcriptome analysis. Application of $B C R-A B L \mathrm{tSS} 2$ to haematopoietic

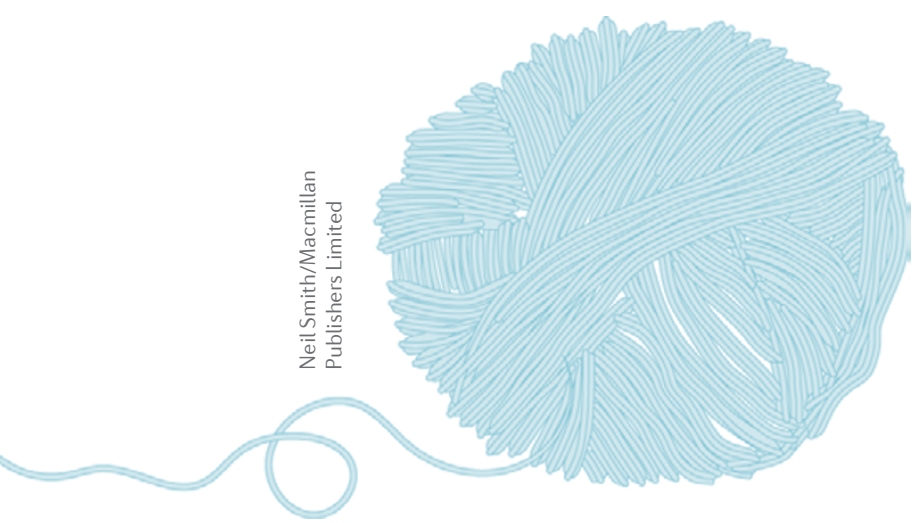

SCs (HSCs; Lin $^{-} \mathrm{CD} 34^{+} \mathrm{CD} 38^{-}$cells)

from a patient treated with a TKI who had CML in haematological remission identified $B C R-A B L$ in single cells, and the method worked as well as did fluorescence in situ hybridization. Furthermore, whole-transcriptome analysis identified genes that were differentially expressed in $B C R-A B L^{+}$compared with non-CML $B C R-A B L^{-}$SCs.

Expanding their analysis, the authors used $B C R-A B L$ tSS2 to examine 2,070 $\mathrm{Lin}^{-} \mathrm{CD} 34^{+} \mathrm{CD} 38^{-}$ bone marrow SCs from diagnosis samples of 20 patients with chronic phase CML (CP-CML). In these samples, $B C R-A B L^{+} \mathrm{SCs}$ had increased expression of genes associated with proliferation and reduced expression of quiescence genes compared with normal HSCs and $B C R-A B L^{-}$SCs. The authors also uncovered several additional differences in gene expression between $B C R-A B L^{+}$and $B C R-A B L^{-}$SCs; analysis of $B C R-A B L^{-}$SCs could potentially identify cell-extrinsic effects of CML on the haematopoietic compartment.

Further analysis of the 16 patients for whom TKI response data were available indicated that although $B C R-A B L^{+}$SCs at diagnosis did not differentially cluster in good versus poor responders, $B C R-A B L^{-} \mathrm{SCs}$ did. At diagnosis, patients who later had a poor response to TKIs had $B C R-A B L^{-}$SCs that expressed genes associated with inflammation, transforming growth factor- $\beta$ (TGF $\beta$ ) and tumour necrosis factor (TNF), and in those who had a good response, both $B C R-A B L^{+}$and $B C R-$ $A B L^{-}$SCs expressed genes associated with proliferation. This suggests that at early stages, patients who are likely to develop TKI resistance have SCs that exhibit properties of quiescence.
In patients who had commenced TKI therapy, the number of $B C R-A B L^{+}$ $S C s$ was reduced post-treatment. In addition, patients who achieved at least haematological remission had two clusters of $B C R-A B L^{+} S C$ s: one enriched for quiescence-associated genes, and one enriched for proliferation-associated genes. More prolonged TKI treatment or achievement of a major molecular response increased the number of quiescent $B C R-A B L^{+}$SCs. These quiescent cells also had enrichment of TGF $\beta$, TNF and WNT- $\beta$-catenin-associated gene expression relative to normal HSCs; these pathways might represent targets for therapeutic elimination of these residual $B C R-A B L^{+} S C s$.

Three patients had progressed from CP-CML to blast crisis (BC)CML. Comparison of $B C R-A B L^{+}$ SCs in these patients at $\mathrm{CP}$ and $\mathrm{BC}$ revealed a distinct cluster of cells. In one patient with lymphoid BC, the authors were able to identify two groups of $B C R-A B L^{+} S C$ s in the diagnosis sample - one clustering close to the $\mathrm{BC}$ cells and one closer to $\mathrm{CP}$ cells. This shows the evolution of the disease in this patient, and provides early evidence of later BC progression. This patient also had a somatic mutation in RUNX1 in the BP-SC cluster, which might have driven $B C$ transformation.

This technique has begun to provide insights into mechanisms of therapeutic resistance and evolution in CML, and has the potential to be applied to other malignancies, although some of these may present greater technical challenges.

Sarah Seton-Rogers

ORIGINAL ARTICLE Giustacchini, A.,

Thongjuea, S. et al. Single-cell transcriptomics uncovers distinct molecular signatures of stem cells in chronic myeloid leukemia. Nat. Med. http://dx.doi.org/10.1038/nm.4336 (2017) 